\title{
Neural Coding Mechanisms in Tactile Pattern Recognition: The Relative Contributions of Slowly and Rapidly Adapting Mechanoreceptors to Perceived Roughness
}

\author{
David T. Blake, Steven S. Hsiao, and Kenneth O. Johnson \\ Krieger Mind/Brain Institute, Neuroscience Department, and Biomedical Engineering Department, Johns Hopkins \\ University, Baltimore, Maryland 21218
}

\begin{abstract}
Tactile pattern recognition depends on form and texture perception. A principal dimension of texture perception is roughness, the neural coding of which was the focus of this study. Previous studies have shown that perceived roughness is not based on neural activity in the Pacinian or cutaneous slowly adapting type II (SAII) neural responses or on mean impulse rate or temporal patterning in the cutaneous slowly adapting type I (SAl) or rapidly adapting (RA) discharge evoked by a textured surface. However, those studies found very high correlations between roughness scaling by humans and measures of spatial variation in SAl and RA firing rates. The present study used textured surfaces composed of dots of varying height (280-620 $\mu \mathrm{m})$ and diameter $(0.25-2.5 \mathrm{~mm})$ in psychophysical and neurophysiological experiments. RA responses were affected least by the range of dot diameters and heights that produced the widest variation in perceived roughness, and these responses
\end{abstract}

could not account for the psychophysical data. In contrast, spatial variation in SAI impulse rate was correlated closely with perceived roughness over the whole stimulus range, and a single measure of SAI spatial variation accounts for the psychophysical data in this (0.974 correlation) and two previous studies. Analyses based on the possibility that perceived roughness depends on both afferent types suggest that if the $\mathrm{RA}$ response plays a role in roughness perception, it is one of mild inhibition. These data reinforce the hypothesis that SAI afferents are mainly responsible for information about form and texture whereas RA afferents are mainly responsible for information about flutter, slip, and motion across the skin surface.

Key words: pattern recognition; texture; roughness; mechanoreceptor; somatosensory; neurophysiology; psychophysics; rhesus
The issue addressed in this paper is the neural code underlying tactile roughness perception. The possibilities include intensive, temporal, or spatial coding mechanisms in any of the four cutaneous mechanoreceptive afferent populations that innervate the hand. Texture perception and its neural mechanisms have been studied extensively (Lederman, 1974; LaMotte, 1977; Lederman et al., 1982; Johnson, 1983; Sathian et al., 1989; Phillips et al., 1992; Phillips and Matthews, 1993; Burton and Sinclair, 1994; Johnson and Hsiao, 1994). The earliest studies provided quantitative characterization of roughness perception and demonstrated that all cutaneous mechanoreceptive afferents except cutaneous slowly adapting type II (SAII) afferents (Phillips et al., 1992) respond vigorously to textured surfaces and could provide a basis for roughness perception. Two combined psychophysical and neurophysiological studies (Connor et al., 1990; Connor and Johnson, 1992) have since narrowed the coding possibilities to those based on spatial variation in cutaneous slowly adapting type I (SAI) and rapidly adapting (RA) impulse rates. The study reported here was designed to investigate the relative contributions of SAI and RA afferents to roughness perception.

The two previous studies in our laboratory (Connor et al., 1990; Connor and Johnson, 1992) used a two-part strategy which

\footnotetext{
Received March 12, 1997; revised July 10, 1997; accepted July 14, 1997.

This study was supported by National Institutes of Health Grants NS18787 and NS34086 and by the W. M. Keck Foundation. We thank John Lane, Hai Dong, David O'Shaughnessy, and Steve Patterson for their assistance.

Correspondence should be addressed to Dr. Kenneth O. Johnson, 338 Krieger Hall, Johns Hopkins University, 3400 North Charles Street, Baltimore, MD 21218. Copyright (C) 1997 Society for Neuroscience $0270-6474 / 97 / 177480-10 \$ 05.00 / 0$
}

we continue in the present study. First, both studies used stimulus surfaces composed of raised dots of varying spacing and diameter that would affect the intensive, temporal, and spatial aspects of the neural population response so differently that only one or a subset of the candidate neural coding possibilities could account for the observed psychophysical behavior. Second, they used consistency as the test of a neural coding hypothesis, only rejecting a candidate neural code when it could be shown that there is no consistent relationship between that coding measure and the psychophysical magnitude judgments. The first study (Connor et al., 1990) used patterns with different dot spacings and diameters to rule out codes based on neural activity in the Pacinian (PC) response as well as codes based on mean impulse rate in the SAI and RA population responses. The second study (Connor and Johnson, 1992) manipulated dot spacing in a different way to rule out temporal variation in impulse rate (and mean impulse rate again). As seen in the first study, measures of SAI and RA spatial variation in impulse rates were consistent with the psychophysical responses over the whole set of stimulus surfaces.

The present study uses raised dot patterns with constant dot spacing but varying dot height and diameter to differentiate the roles of SAI and RA afferents. A companion study (Blake et al., 1997) showed that SAI but not RA impulse rates are affected strongly by changes in pattern height. Those results were used to design surfaces that range widely in perceived roughness and that affect SAI and RA responses differently. Combined psychophysical and neurophysiological studies with these surfaces show that roughness magnitude is accounted for entirely by SAI responses and that a single, simple, physiologically plausible neural coding 
hypothesis accounts for the psychophysical behavior in all three studies. When the finger pad is directly in contact with a surface, roughness perception depends on the firing rates of a population of central neurons sensitive to differences in firing rates between SAI afferent fibers innervating skin regions separated by 1-3 $\mathrm{mm}$. Analyses are presented that suggest that the RA role, if any, is one of mild inhibition.

\section{MATERIALS AND METHODS}

Stimulus surfaces. Stimuli were fabricated from plastic sheets with a photosensitive layer that is water-soluble until exposed to UV light (Toyoba Printight Plastics, EF series). Photographic negatives of the stimulus patterns were laid over the sheets before UV exposure so that only the material constituting the raised pattern would be polymerized. The thickness of the photosensitive layer determined the height of the raised patterns. The thicknesses used in this study were those that were commercially available $(280,370$, and $620 \mu \mathrm{m})$ and nearest to the heights that were desired for the study. After exposure, the unexposed portions of the surface layer were scrubbed away lightly in warm water, and the plastic was dried and exposed to UV light again. Three surfaces differing only in dot height $(280,370$, and $620 \mu \mathrm{m})$ were composed of dots arranged in tetragonal arrays with constant center-to-center spacings of $3.5 \mathrm{~mm}$ (see the pattern illustrated in Fig. $3 A$ ). The dots were truncated cones with flat tops and sides sloping at $60^{\circ}$ relative to the plane of the surface. The surfaces, $20 \mathrm{~mm}$ wide $\times 220 \mathrm{~mm}$ long, were attached to the circumference of a cylindrical drum that was used in both the psychophysical and the neurophysiological experiments. Within each surface, dot diameters (measured at the tops of the truncated cones) increased at a uniform rate from $0.25 \mathrm{~mm}$ at one end to $2.50 \mathrm{~mm}$ at the other end.

Psychophysical methods. Subjects inserted the hand used for writing through a screen and rested the hand on a flat plate, with the distal pad of the index finger over a $25 \times 25 \mathrm{~mm}$ square aperture. On each trial, the drum with the stimulus surfaces was translated, rotated, and stopped so that a portion of a single surface was beneath the subject's finger. Translation along the axis of the drum selected one of the three dot heights, and rotation selected one of the six dot diameters used in the experiment. Because the dot diameters varied continuously in one direction along the pattern, the dot diameters varied by $\pm 14 \%$ of the nominal spacing within the $25 \times 25 \mathrm{~mm}$ region exposed to the subject; for example, when the dots $1.15 \mathrm{~mm}$ in diameter were presented, the dot diameters within the scanning region ranged from 0.99 to $1.31 \mathrm{~mm}$. Subjects typically avoided the edges of the aperture and contacted only the central two-thirds of the exposed region. Contact force was controlled by mounting the drum on a counterbalanced beam, weighted so that the drum pressed against the plate with a force of $100 \mathrm{gm}$. Subjects were instructed to depress the surface slightly during palpation, ensuring a contact force of $100 \mathrm{gm}$. This resulted in an oval contact region averaging $12 \mathrm{~mm}$ wide $\times 15 \mathrm{~mm}$ long and in an average contact pressure of $0.7 \mathrm{gm} / \mathrm{mm}^{2}$. Subjects scanned each surface with distal to proximal movements, raising the finger during extension to ensure scanning in only one direction. This caused the textured surface to move distally over the skin as was done in the neurophysiological experiments. After scanning the surface for up to $10 \mathrm{sec}$, subjects were required to respond with a number proportional to perceived roughness.

Roughness was not defined for the subjects; instead, they were told to use their own concept of roughness from daily experience and to estimate roughness magnitude as a number proportional to the strength of their perception of roughness using any numerical range that seemed appropriate. At the outset of the experiment, five representative surfaces requiring no response were presented to familiarize the subjects with the surfaces. Each subject was then presented with 18 different surfaces made up of tetragonal arrays of dots that were combinations of one of six dot diameters $(0.25,0.70,1.15,1.60,2.05$, or $2.5 \mathrm{~mm})$ and one of three dot heights $(280,370$, or $620 \mu \mathrm{m})$. Each surface was presented once in each of four randomized blocks of 18 trials. The psychophysical data from each subject were normalized by dividing each numerical response by the mean of all 72 responses for the subject. The normalized values were averaged within subjects to produce a value for each surface and then across subjects to produce a grand mean for each of the 18 surfaces.

Neurophysiological methods. Experiments were conducted on barbiturate-anesthetized rhesus monkeys that weighed between 3.0 and $5.0 \mathrm{~kg}$. Single cutaneous mechanoreceptive fibers were dissected from the median or ulnar nerves using methods described previously (Talbot et al.,
1968). Afferent fibers were classified as SAI, RA, or PC on the basis of responses to a vibrating point probe (Talbot et al., 1968). Only SAI and RA afferents with receptive fields on the distal pads of digits $2-5$ were studied. After such an afferent was found, the surface of the drum bearing the stimulus pattern (Johnson and Phillips, 1988) was lowered onto the skin and adjusted so the center of the receptive field was aligned with the center of the contact patch. The stimulus surface was scanned across the finger pad with a controlled force of $30 \mathrm{gm}$ and with a constant proximal-to-distal scanning velocity of $40 \mathrm{~mm} / \mathrm{sec}$. That $30 \mathrm{gm}$ force produced an oval contact region on the monkey finger pad that averaged $7.0 \mathrm{~mm}$ wide $\times 9.0 \mathrm{~mm}$ long and had an average contact pressure of 0.6 $\mathrm{gm} / \mathrm{mm}^{2}$. The contact pressure and scanning velocity were chosen to be similar to those used by humans in roughness estimation experiments when force and velocity are unconstrained (Lederman, 1974). The exact velocity is not critical because roughness estimation has been shown to be unaffected by changes in scanning velocity within the range of $10-50$ $\mathrm{mm} / \mathrm{sec}$ (Lederman, 1974).

After each rotation, the drum was shifted $0.2 \mathrm{~mm}$ in the axial direction (at right angles to the direction of rotation). This was repeated until the entire pattern was scanned across the distal finger pad. During the experiment, the occurrence times of action potentials and stimulus position signals were recorded with an accuracy of $0.1 \mathrm{msec}$. A shaft encoder mounted on the drum allowed us to track drum position with a precision of $8 \mu \mathrm{m}$ (Johnson and Phillips, 1988).

The occurrence times of action potentials and stimulus position indicators were used to construct a two-dimensional spatial event plot (SEP), in which each action potential was assigned $x$ and $y$ coordinates corresponding to the position of the stimulus pattern when the action potential occurred. Then the SEPs were converted into two-dimensional firing rate arrays of $0.2 \times 0.2 \mathrm{~mm}$ bins with a two-dimensional, adaptive Parzen estimator (Twombly et al., 1996), which replaces each impulse with a two-dimensional Gaussian function with unity volume. The ratio and orientation of the Gaussian major and minor axes are determined by the covariance of the locations of all impulses within $1.0 \mathrm{~mm}$ of the target impulse in the SEP. The spread (SD of the major axis) is scaled so that it is proportional to the inverse of the square root of the number of impulses within this $1.0 \mathrm{~mm}$ radius. Thus, an impulse in a region of high firing rate is represented by a tall, narrow Gaussian function, whereas an isolated impulse is represented by the widest allowable Gaussian function, which, in this application, was a circular distribution with an SD equal to $0.63 \mathrm{~mm}$ in all directions. Then, the Gaussian volume overlying each $0.2 \times 0.2 \mathrm{~mm}$ bin was calculated to generate a two-dimensional array of firing rates. This binning method was used because extensive analyses with simulated spike trains showed that this method generates impulse rate estimates with lower SEs than conventional bucket binning or fractional interval binning generate. The two-dimensional arrays of firing rates were used to compute the response areas, mean firing rates, and spatial variation in firing rate.

Analysis of spatial variation. Spatial variation in impulse rate has been found to closely match psychophysical estimates of roughness magnitude in two previous studies (Connor et al., 1990; Connor and Johnson, 1992). Therefore, spatial variation in impulse rate was computed with twodimensional Gabor filters as described by Connor and Johnson (1992). A Gabor filter is a mathematically defined function that is sensitive to local periodicity in firing rate. The following formula was used for computing the Gabor filter:

$$
f(x, y)=\frac{1}{\sqrt{\left(2 \pi \sigma^{2}\right)}} e^{-\left(x^{2}+y^{2}\right) / 2 \sigma^{2}} \sin (2 \pi(x \cos (\theta)+y \sin (\theta)) / \lambda+\phi),
$$

where $x$ and $y$ specified location relative to the center of the filter ( $x$ is along the scanning direction), $\sigma$ specified the SD of a symmetric twodimensional Gaussian envelope that specified the local area over which spatial variation in impulse rate was computed, $\theta$ specified the orientation of the sinusoidal component of the filter, $\lambda$ specified the spatial wavelength of the sinusoid, and $\phi$ specified the phase of the sinusoid relative to the center of the filter. Gabor filters are most sensitive to firing-rate differences separated by a distance $\lambda / 2$ in the direction of sinusoidal variation.

Each Gabor filter extracts the spatial variation in impulse rates at a given spatial frequency, area, phase, and orientation. The correlation between perceived roughness and this measure of spatial variation in the work by Connor and Johnson (1992) was independent of phase and orientation; thus, the rate variation measure was averaged across four phases $(\phi=0, \pi / 2, \pi$, and $3 \pi / 2)$ and six orientations $\left(\theta=0-150^{\circ}\right.$ in $30^{\circ}$ 
increments). Spatial variation in the two-dimensional firing rate arrays was computed by selecting a set of filter values, $\lambda$ and $\sigma$, and convolving the Gabor filters with the input array. The convolution formula was:

$$
\operatorname{output}(x, y)=\sum_{u} \sum_{v} \operatorname{input}(x+u, y+v) f(u, v) \Delta u \Delta v
$$

where input was the two-dimensional firing-rate array, $f$ was the selected Gabor filter, $\Delta x$ and $\Delta y$ were the bin sizes in the $x$ and $y$ directions, and $u$ and $v$ varied from $-\lambda$ to $+\lambda$ in $0.2 \mathrm{~mm}$ increments. When the wavelength was small compared with the width of the Gaussian envelope $(\lambda<2 \sigma), u$ and $v$ varied from $-1.5 \lambda$ to $+1.5 \lambda$.

The convolution was computed over each neural record, which consisted of all the responses of an afferent to one dot height (for a typical record, see Fig. $3 B$ ). In the neural record, the analysis region for a given dot diameter was centered in the neural responses to that dot diameter. The analysis region (the range of $x$ and $y$ ) was $14.8 \mathrm{~mm}$ long (three cycles of the stimulus pattern in the scanning direction). The width of the analysis region was set to two cycles of the stimulus pattern $(9.8 \mathrm{~mm})$ except for the few cases when there were too few scans to provide two full cycles; then, only one cycle $(5.0 \mathrm{~mm})$ was used. The analysis region was an integral number of stimulus cycles wide and long in all cases and was always surrounded by sufficient data to make convolution over the entire region possible; that is, data always extended at least $u$ and $v$ units beyond the $x$ and $y$ edges of the analysis region. This analysis region was approximately the same as the contact region in the psychophysical experiments.

Because the Gabor filter includes equal negative and positive weights, convolution without rectification produces a value that is, on average, zero regardless of the spatial variation in firing rates. Rectification is required to obtain a measure that is proportional to this spatial variation. The process of convolution, rectification, and averaging was repeated for each of the orientations and phases of the Gabor filters. The results of all these convolutions were averaged to achieve the overall spatial variation in firing rates for the given afferent at each dot diameter and height. The final SAI or RA spatial variation values were found by averaging across neurons of the same type. The measure spatial variation in impulse rate has the same units, impulses per second (ips), that the input data have, because the Gabor weights are dimensionless.

The best parameters for the Gabor filters were determined for each afferent type by iterative adjustment of the wavelength and SD to maximize the correlation with the psychophysical data. Gabor wavelengths ranging from 2.0 to $5.0 \mathrm{~mm}$ and SDs ranging from 10 to $100 \%$ of the wavelength were used. Only one local maximum was found in the total range of parameters tested for SAI or RA afferents.

Response area and firing rate. Response area was measured with an algorithm designed to yield areal measures that are consistent with but less variable than the measures used by Johnson and Lamb (1981) and Phillips et al. (1992). Those investigators measured the single-dot response area by finding the maximal firing rate in any $0.2 \times 0.2 \mathrm{~mm}$ bin in the response area and then by including a bin in the response area if its firing rate exceeded $10 \%$ of the maximal firing rate. In the procedure used here, the mean firing rate across a circular area $1 \mathrm{~mm}$ in diameter was determined for every position in the analysis region, and the maximum of these mean firing rates was used. This measure was, on average, $40 \%$ of the maximum firing rate measure used by Phillips et al. (1992), but its coefficient of variation was $<10 \%$ when applied to different dots of the same diameter and height in the same neural record. Area was calculated by counting bins whose firing rate exceeded $25 \%$ of the new maximum rate. The mean firing rate over the response area was the mean rate of all bins included in the response area.

\section{RESULTS}

Psychophysical data were obtained from 15 subjects (11 male, 4 female; ages 22-30 years) who reported the roughness of 18 surfaces composed of raised dots in tetragonal arrays with constant center-to-center spacings of $3.5 \mathrm{~mm}$ (see Fig. 3). The surfaces differed only in dot height $(280,370$, and $620 \mu \mathrm{m})$ and diameter $(0.25,0.70,1.15,1.60,2.05$, and $2.50 \mathrm{~mm})$. Neurophysiological data were obtained from $16 \mathrm{SAI}$ and $16 \mathrm{RA}$ afferents in five rhesus monkeys using the same surfaces that were used in the psychophysical study.

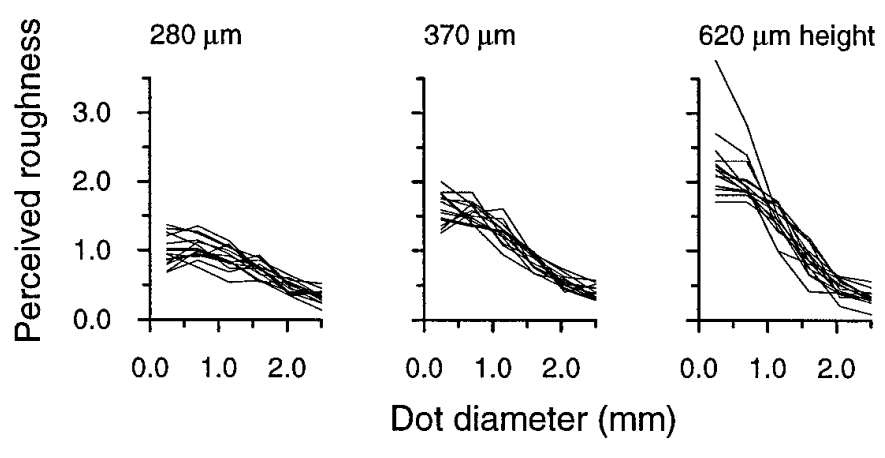

Figure 1. Psychophysical results for individual subjects. Each subject reported the roughness of each surface four times in randomized blocks of trials. All numerical reports from a single subject were normalized to a mean of 1.0 to eliminate individual differences in the range of numbers chosen for ratio scaling. Each line shows the averaged responses of a single subject. Stimuli had dot diameters of $0.25,0.70,1.15,1.60,2.05$, and 2.50 $\mathrm{mm}$ and heights of 280,370 , and $620 \mu \mathrm{m}$.

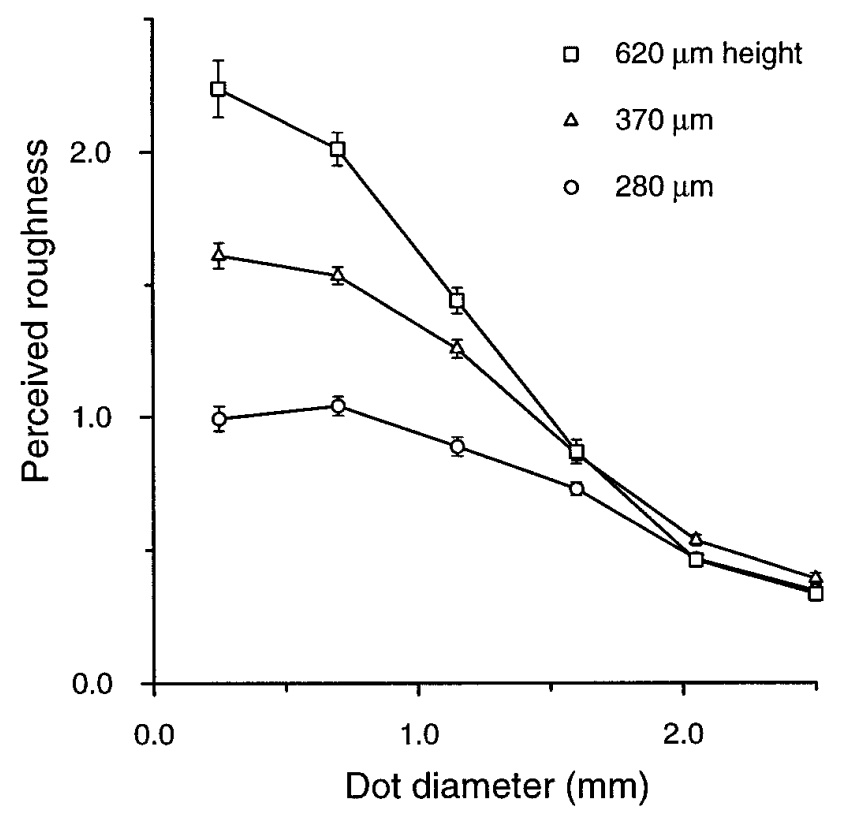

Figure 2. Psychophysical averages. Each point represents the mean roughness report across all subjects for one stimulus surface. Error bars represent 1 SEM.

\section{Psychophysical results}

The mean subjective response of each subject to each combination of dot height and diameter is shown in Figure 1, which illustrates the consistency between subjects. The mean perceived roughness for each surface, averaged across subjects, is shown in Figure 2. The roughest surfaces were described as very rough, and the least rough surfaces were described as moderately smooth. The magnitude judgments shown in Figure 2 span a 7-1 range. The effects of dot height and width were both significant, and there were significant interactions between the effects (two-way ANOVA, $p<0.001$ ), as can be seen in Figure 2. At small diameters, dot height had a large, almost linear effect on subjective roughness. At diameters of 2.0 and $2.5 \mathrm{~mm}$, dot height had no significant effect on reported roughness.

\section{Neurophysiological results}

The responses of SAI and RA afferents to the dot patterns were similar (Fig. 3). Both afferent types responded to narrow dots 


\section{A. Stimulus pattern}

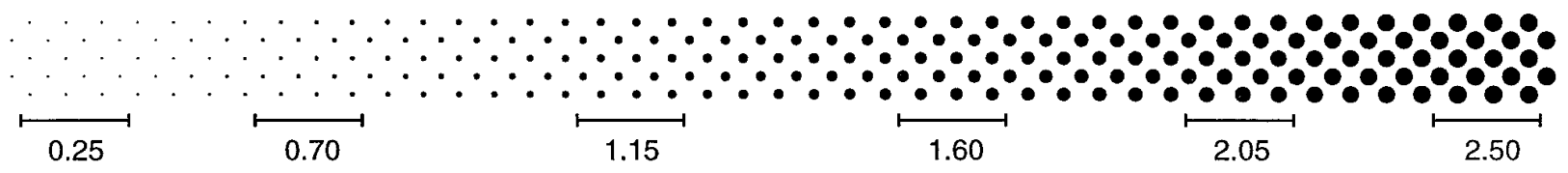

\section{B. Neuronal responses}

\section{Dot \\ Height
$(\mu \mathrm{m})$}

\section{SAI}

280
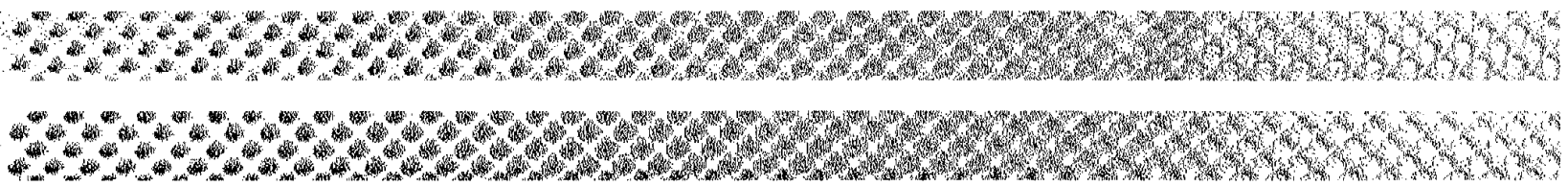

620

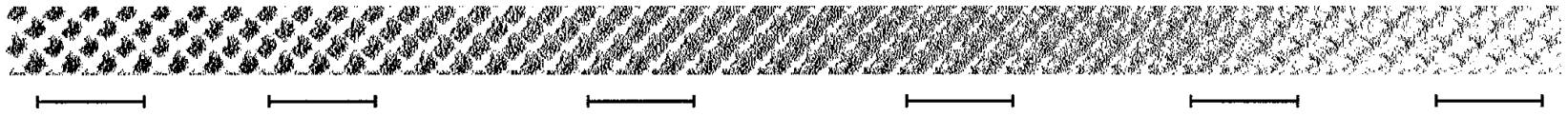

RA

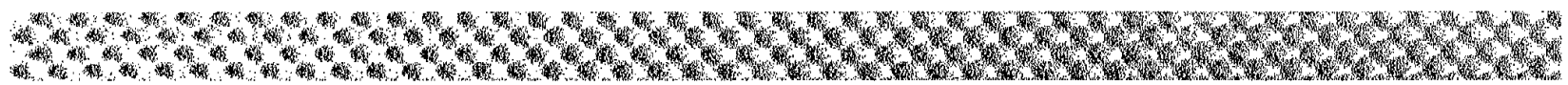

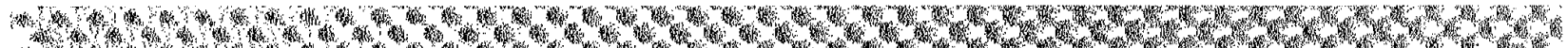
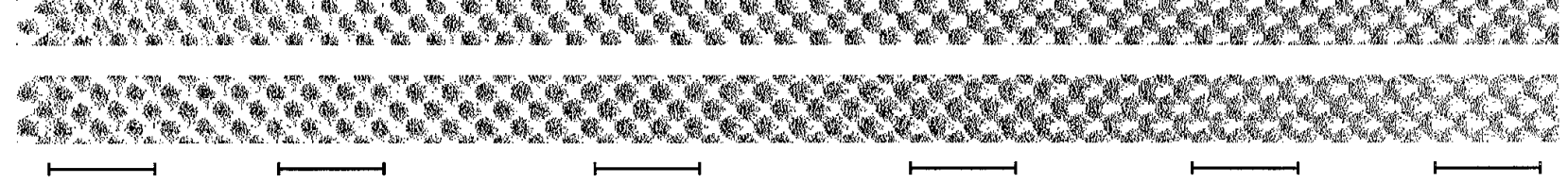

Figure 3. Stimulus pattern and typical SAI and RA responses. $A$, The stimulus surfaces were tetragonal arrays of raised dots arranged in strips, 20 mm wide $\times 220 \mathrm{~mm}$ long, around the circumference of a cylindrical drum. Dot diameters increased uniformly from 0.25 to $2.5 \mathrm{~mm}$ along the length of each pattern. The center-to-center dot spacing was constant at $3.5 \mathrm{~mm}$. Only dot height differed between surfaces. The regions used for psychophysical testing are indicated by bars below the stimulus pattern. $B$, Typical SAI and RA afferent responses to the same stimulus patterns used in the psychophysical experiments. Each tick mark in the raster represents an action potential and is located at the position in the stimulus pattern at which it occurred. The bars below the responses mark the analysis regions, which were $14.8 \mathrm{~mm}$ long and correspond to the locations used in the psychophysical experiments.

with localized bursts of activity; the activity became weaker and less localized as dot diameter increased (and the gap between dots decreased). When dot diameter exceeded $2.0 \mathrm{~mm}$, the activity was localized to the edges of the dots. The primary difference between the SAI and RA responses (Fig. 3) is that the SAI responses to dots with small diameters were strongly affected by variations in dot height whereas the RA responses were insensitive to those same variations. This difference between the two afferent types is also shown in Figure 4, in which examples of rate profiles evoked by dots of different heights are presented. The SAI response to a dot $2.05 \mathrm{~mm}$ in diameter is confined to a single narrow peak because this afferent responded to only one edge of the dot. A similar response is illustrated in Figure 3.

The SAI responses paralleled the psychophysical results qualitatively. As shown in the psychophysical experiments, dot height had a major effect on SAI impulse rates at dot diameters of $<2.0$ $\mathrm{mm}$. At diameters of $>2.0 \mathrm{~mm}$, dot height had little, if any, effect on the psychophysical responses or on the responses of the SAI afferents. In contrast, the RA responses were largely, or wholly, unaffected by dot height at any diameter. Quantitative analyses confirm these observations. Simple quantitative analyses of the neural responses were restricted to the patterns with dot diameters of $<1.5 \mathrm{~mm}$, because most of the psychophysical effects of dot diameter and height were restricted to these surfaces. The data illustrated in Figures 3 and 4 suggest that the spatial and intensive

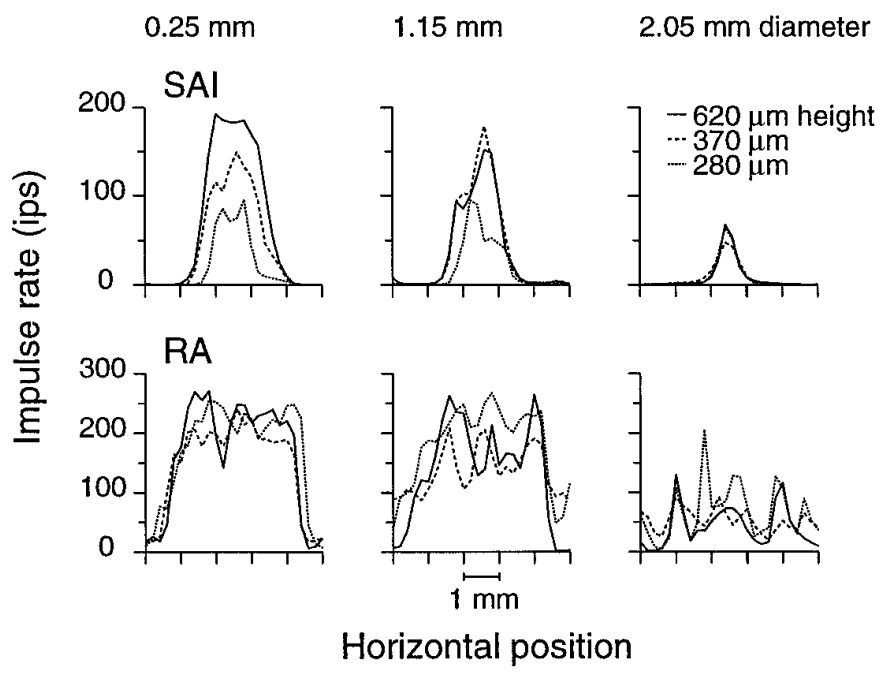

Figure 4. Impulse rate profiles evoked by single dots from a typical neuron. Each plot shows the firing rate when dots passed through the center of the receptive field. The left, middle, and right plots show typical SAI and RA responses to narrow, intermediate, and wide dot diameters, respectively. 

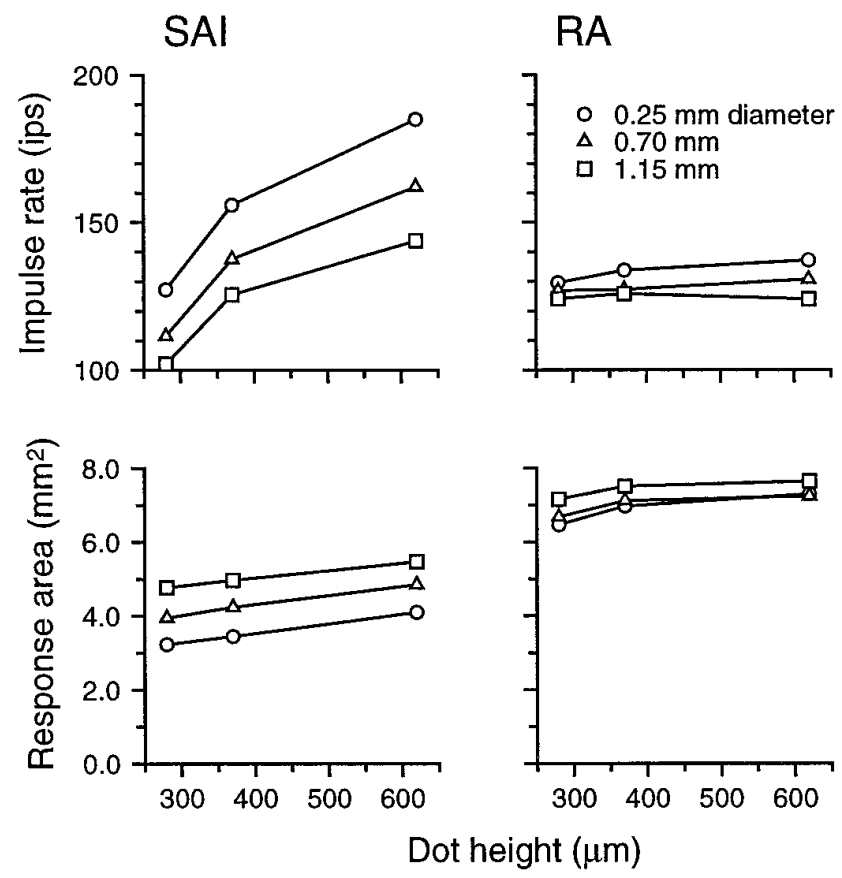

Figure 5. SAI and RA mean rate (top) and response area (bottom) versus dot height and diameter. Response area is defined here as the area of skin over which a single scanned dot evokes a discharge rate of $>10 \%$ of the peak discharge rate (see Materials and Methods). Impulse rate is the mean firing rate within this area. The SEs for SAI and RA response areas ranged from 0.48 to $0.81 \mathrm{~mm}^{2}$ and from 0.97 to $1.298 \mathrm{~mm}^{2}$, respectively. The SEs for SAI and RA mean impulse rates ranged from 9.8 to 13.5 ips and from 4.0 to $5.9 \mathrm{ips}$, respectively.

structure of the responses to single dots with diameters of $<1.5$ $\mathrm{mm}$ can be described by simple measures of mean rate and response area (see Materials and Methods). Figure 5 shows that RA responses were driven strongly but were almost completely unaffected by changes in dot diameter and height. Although there is a suggestion of a small drop in RA firing rate and response area at the smallest dot height, the effect, if any, is not close to statistical significance (two-way ANOVAs of impulse rate and response area vs dot height and diameter, $p>0.1$ ). In contrast, SAI responses, particularly mean impulse rate, were affected strongly by both dot height and diameter (two-way ANOVA, $p<$ 0.001 and $p<0.005$ for effects of height and diameter on rate and on area, respectively). SAI firing rates increased as dot height increased or dot diameter decreased. SAI afferent response area increased as dot diameter or height increased. These results show that RA afferent responses cannot account for the psychophysical responses to the nine surfaces with dot diameters of $<1.5 \mathrm{~mm}$, which leaves only candidate codes for roughness based on SAI responses. The wide range of SAI responses illustrated in Figure 5 matches qualitatively the wide range of psychophysical roughness estimates evoked by the nine surfaces with the three smallest diameters (Fig. 2).

\section{Neural coding}

A fundamental requirement of any neural coding hypothesis is consistency (Connor et al., 1990; Johnson et al., 1996). If it is hypothesized that a single perceptual dimension, $P$, depends on a neural code, $N$, then there must be a one-to-one relationship between $N$ and $P$. If two or more stimuli yield the same neural coding value but significantly different perceptual intensities, then
$N$ is not the basis for $P$. It may be a component of the neural code, but it is not by itself an adequate specification of the code.

An important point is that we rely on data from previous studies as well as the data from this study to exclude all possible neural bases for roughness perception except one. If the data presented here had to be considered in isolation, we could not convincingly exclude the possibility that roughness perception is based on mean impulse rate in the SAI population responses (compare Figs. 2 and 5). However, the psychophysical and neural responses to textured surfaces used in our two previous studies (Connor et al., 1990; Connor and Johnson, 1992) showed that there is no consistent relationship between any measure of firing rate and psychophysical roughness judgments. The second study (Connor and Johnson, 1992) eliminated temporal coding measures as possible neural codes (see Discussion) and showed that only measures of spatial variation in the firing rates of either SAI or RA afferents could account for the findings of the first two studies. So, the surfaces used in the current study were designed to distinguish between SAI and RA roles. They were not designed to distinguish between intensive, temporal, and spatial mechanisms. We rely on previous studies for the exclusion of all possibilities except SAI and RA spatial variation in impulse rate as the basis for roughness perception. Three possibilities were tested in the present study: that the roughness judgments displayed in Figures 1 and 2 were based on spatial variation in the SAI firing rates, spatial variation in the RA firing rates, or some combination of the two.

Plots of spatial variation in the SAI and RA impulse rates measured with Gabor filters (see Materials and Methods) are illustrated in Figure 6. A comparison of the data in Figures 2 and 6 shows that spatial variation in the SAI population response accounts well for the psychophysical magnitude estimation functions. At dot diameters below $1.5 \mathrm{~mm}$, dot height had a significant effect on both the SAI spatial variation and the psychophysical results. In contrast, the spatial variation in RA rate was affected little by changes in dot height, as expected from the analyses shown in Figure 5. The decline in SAI and RA spatial variation at dot diameters $>1.5 \mathrm{~mm}$ comes from the rapid decline in mean impulse rate when the dot diameters approach the center-tocenter spacing between dots.

The plots illustrated in Figure 6 are based on Gabor filters with SDs and wavelengths of 1.0 and $2.6 \mathrm{~mm}$, respectively, for the SAI data and 2.5 and $4.2 \mathrm{~mm}$, respectively, for the RA data. Although these parameters yielded the best fit between the psychophysical and neurophysiological data, the fit was very broad. For example, Gabor wavelengths ranging from 2.0 to $3.2 \mathrm{~mm}$ produced correlations between predicted and observed roughness judgments exceeding 0.96 ; the $2.6 \mathrm{~mm}$ wavelength produced a correlation of 0.974 .

A consistency plot of the reported subjective roughness intensity against the putative neural coding measure is shown in Figure 7. As seen in previous studies (Connor et al., 1990; Connor and Johnson, 1992), deviations from a simple, near-linear relationship between reported roughness and SAI spatial variation in impulse rate were of the same magnitude as the inherent variability of the neural and psychophysical measures themselves. Spatial variation in SAI impulse rates provides an effective, consistent basis for the graded roughness judgments observed in the psychophysical experiments. In contrast, the RA spatial variation measures illustrated in Figure 7 provide no consistent basis for the observed psychophysical behavior. The six points at the lower left of the RA graph of Figure 7, which correspond to the surfaces with the two 

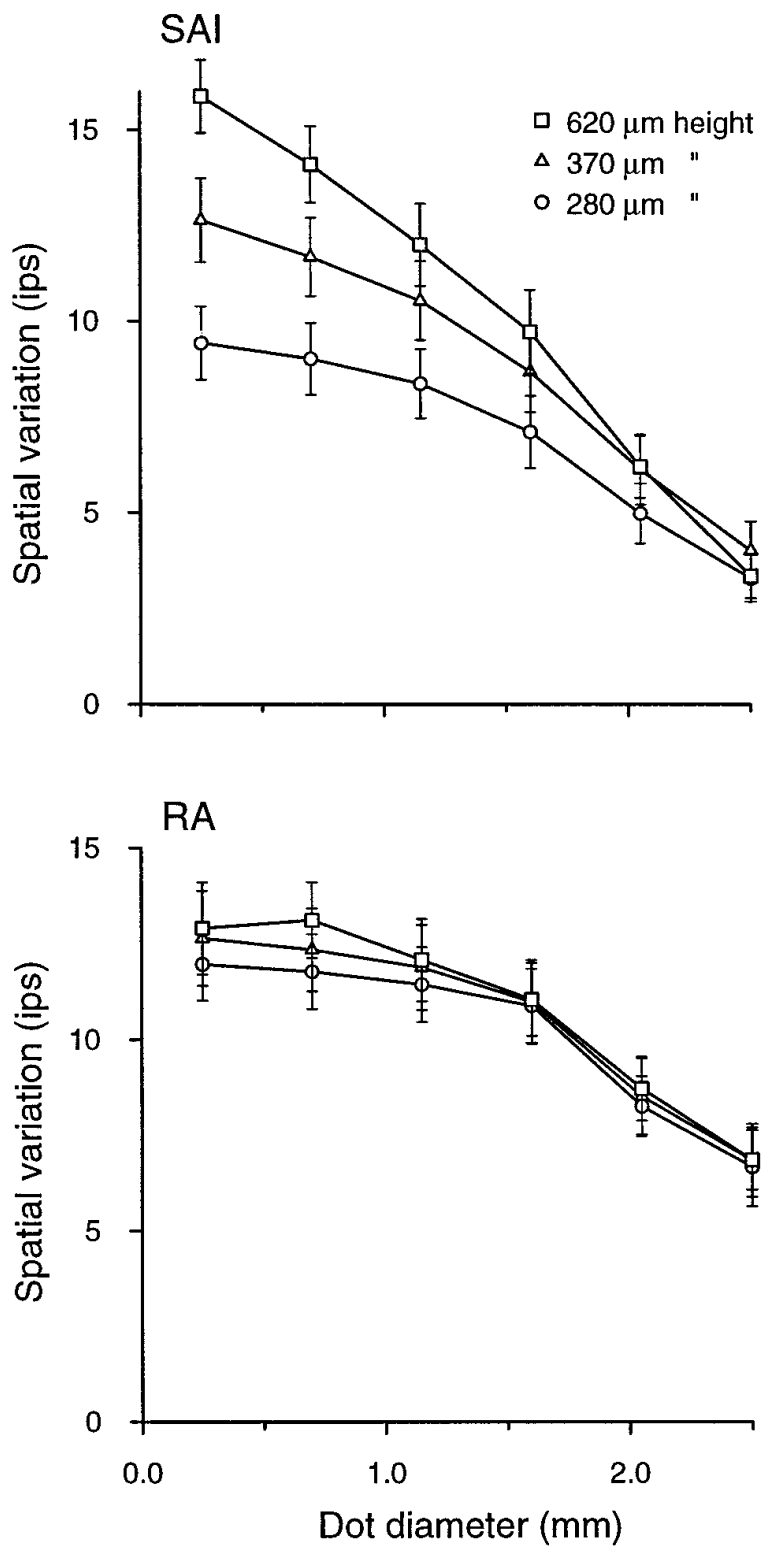

Figure 6. Spatial variation in firing rates versus dot diameter. The measures of spatial variation in firing rates displayed here and in successive figures were obtained by convolving the afferent discharge evoked by a local segment of the stimulus pattern with optimal Gabor filters at all orientations and phases (see Materials and Methods). The SD and wavelength of the Gabor filters used with the SAI data were 1.0 and $2.6 \mathrm{~mm}$, respectively. The SD and wavelength used with the RA data were 2.5 and $4.2 \mathrm{~mm}$, respectively. The plotted points are averages over all $16 \mathrm{SAI}$ and $16 \mathrm{RA}$ afferents studied here. Error bars represent 1 SEM.

largest dot diameters, are consistent with the psychophysical responses (compare Figs. 2 and 6). However, if it was hypothesized that roughness magnitude judgments depend on RA spatial variation, then it would have to be explained how a measure without statistically significant variation can account for roughness magnitude judgments that vary by a factor of three. The rightmost 12 points in the $\mathrm{RA}$ graph of Figure 7 range between 11 and 13 ips, whereas the roughness judgments to which these points correspond range from 0.73 to 2.24 . All measures including mean impulse rate, response area, and spatial variation in impulse rates in the RA responses illustrated in Figures 4 and 5 were
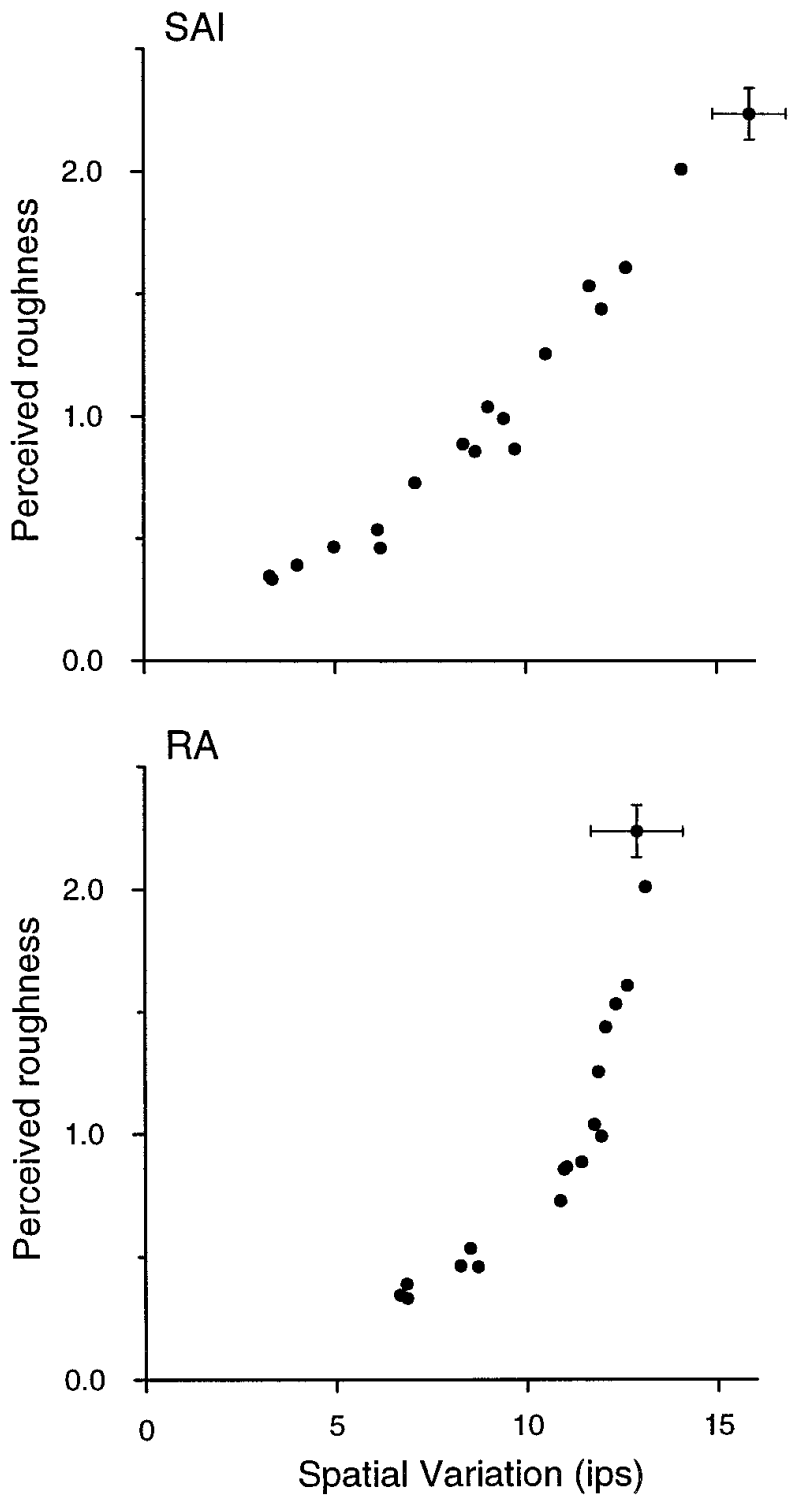

Figure 7. Consistency plots of reported roughness versus SAI and RA spatial variation in afferent firing rates for each of the 18 surfaces. The correlation coefficient between psychophysical data and a Gabor measure of spatial variation in firing rates was 0.974 for SAI afferents and 0.869 for RA afferents. The error bars represent SEMs for the reported roughness and the spatial variation measure associated with the roughest surface in the study (see Figs. 2 and 6).

practically invariant, and no measure provided a consistent basis for the psychophysical responses. Even when the RA spatial variation measure was optimized to match the psychophysical data, it could not provide a consistent basis for two-thirds of the psychophysical observations. Thus we conclude that the roughness judgments must have depended on the SAI responses.

If SAI spatial variation in impulse rate does indeed account for roughness perception, a single measure of spatial variation should account for the psychophysical data from the present study and the previous two studies. Consequently, the SAI neural data from the previous two studies (Connor et al., 1990; Connor and Johnson, 1992) were reanalyzed with the Gabor filter described in this paper. The result, shown in Figure 8, is that a single model based on spatial variation in SAI impulse 

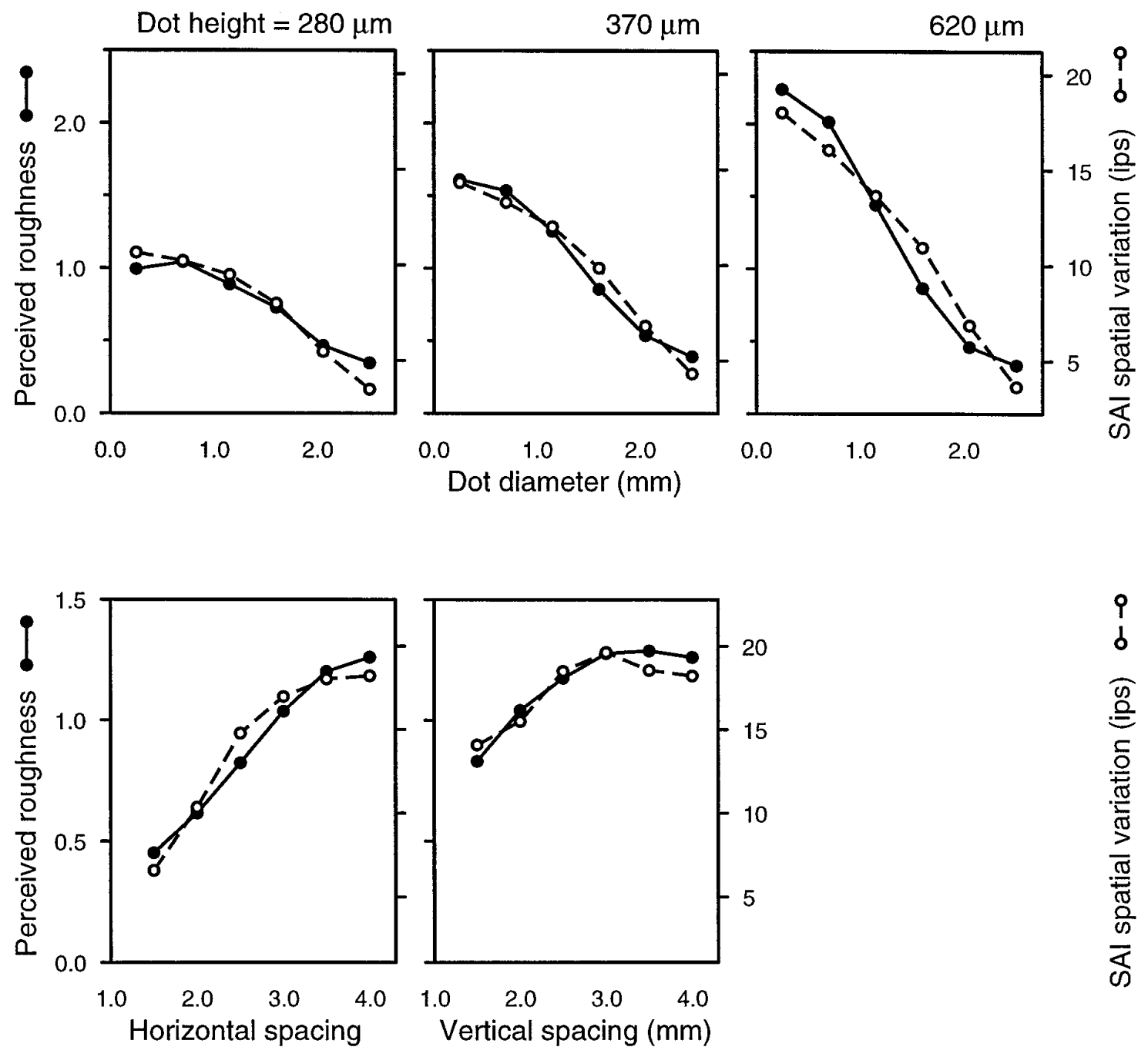

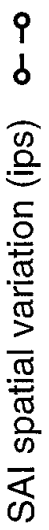

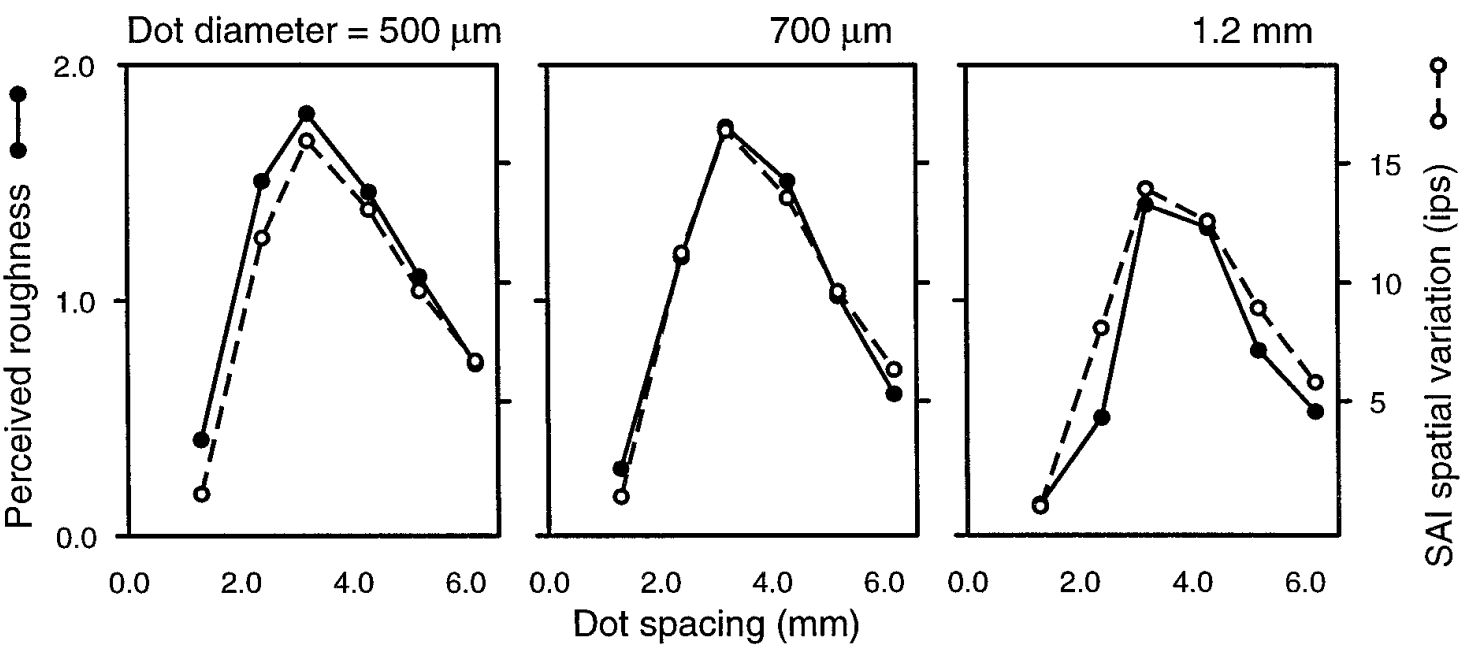

Figure 8. Roughness magnitude and identical measures of spatial variation in SAI firing rates from three studies with different textured surfaces. The neural data from previous studies were reanalyzed using Gabor filters with the same parameters ( $2.6 \mathrm{~mm}$ wavelength; $1.0 \mathrm{~mm}$ SD) as those used in the present study. The left ordinate in each graph is the mean reported roughness. The right ordinate is the associated Gabor measure of spatial variation. The upper panels show the data from the present study. The middle panels show the data from Connor and Johnson (1992), who used 11 dot arrays with varying spacing in either the horizontal or vertical direction and a constant $4.0 \mathrm{~mm}$ spacing in the other direction. The lower panels show data from Connor et al. (1990), who used 18 dot arrays with varying dot spacing and diameter. 
rates alone accounts for the psychophysical behavior in all three studies. Although the Gabor filter formulation allows for the possibility of multiple regions of positive and negative weighting (sinusoidal wavelengths shorter than the width of the filter), the data were best fitted by filters with two main lobes, one positive and one negative, separated by half the sinusoidal wavelength of the filter. Connor and Johnson (1992) found that spatial variation in SAI firing rate measured with a Gabor filter with a wavelength of $2.8 \mathrm{~mm}$ explained their roughness data most effectively. We found that a wavelength of $2.6 \mathrm{~mm}$ per cycle best explained the data from all three data sets. These wavelengths correspond to measures based on spatial differences in firing rate separated by $1.3-1.4 \mathrm{~mm}$. Connor et al. (1990) used a measure of spatial variation based on differences in firing rates between small regions separated by a fixed distance and found that a separation of $2.2 \mathrm{~mm}$ was optimum. The peaks relating goodness-of-fit to separation were broad in all three studies, which indicates that the precise separation is not critical and that the mechanism is not sharply tuned to a specific spatial separation. Thus, our favored hypothesis is that roughness perception is based on spatial variation in the SAI population firing rates at spatial separations between 1 and $3 \mathrm{~mm}$.

Although a single neural coding hypothesis based on spatial variation in the SAI neural image explains $92 \%$ or more of the variance in psychophysical responses from all three studies, the possibility remains that roughness perception depends on a combination of cues from the SAI and RA population responses and that we simply isolated the SAI component in the present experiment. We tested this possibility by performing regression analyses on the psychophysical data from all three studies, using combinations of all the possible neural codes that we have tested. Step-wise regression analysis (SPSS) was performed on all the data from all three studies. In this analysis, putative neural codes are entered and removed one by one until no excluded code increases the explained variance significantly when entered and every included code decreases the explained variance significantly when removed. We included measures of mean impulse rate and spatial and temporal variation in the SAI and RA responses from all three studies and PC measures from the first study. The results from all three studies were consistent. In all cases, SAI spatial variation in impulse rate was entered first, yielding correlation coefficients between predicted and observed roughness magnitudes of $>0.974$. In analyses based on data from the first two studies, spatial variation in RA impulse rate was entered second and contributed significantly, bringing the correlation coefficients between predicted and observed roughness magnitudes above 0.99. However, the regression coefficients specifying the RA role were negative, which suggests that if the RA responses play a role in roughness perception, it is one of inhibition. It should be noted that the improvement in the fit resulting from the inclusion of RA spatial variation was slight in both cases. No other response measures or combination of measures, including Pacinian response measures in the first study, improved the fit. When data from the present study were analyzed, only spatial variation in the SAI impulse rate had a statistically significant effect. When spatial variation in RA impulse rates was forced into the regression, its coefficient was negative, as seen in the first two studies, but the improvement in predicted roughness judgments was not statistically significant (i.e., $p$ was $>0.05$ ).

\section{DISCUSSION}

This is the third in a series of studies aimed at uncovering the neural mechanisms of tactile roughness perception. First, we discuss the cumulative evidence that roughness perception is based on a localized measure of spatial variation in SAI impulse rate. Every other measure of the population response that we have tested has been shown to bear no consistent relationship to roughness perception. Then, we discuss the assumptions linking these studies to the neural mechanisms of roughness perception in humans.

The first of our three studies (Connor et al., 1990) examined spatial, temporal, and intensive neural codes in the SAI, RA, and Pacinian populations as possible bases for roughness perception. That study used surfaces of raised dots of varying dot diameter and spacing. Roughness perception depended strongly on dot spacing (1.3-6.2 mm), peaking at $\sim 3 \mathrm{~mm}$ and declining at smaller and larger spacings. Dot diameter varied over a narrow range $(0.5-1.2 \mathrm{~mm})$ but had the same effect that was seen in the present study, a decline in perceived roughness with increasing dot diameter (Johnson and Hsiao, 1994). These surfaces had two properties that made them effective for testing neural coding hypotheses: (1) roughness perception ranged widely (from "almost perfectly smooth" to "very rough"); and (2) surfaces with widely differing spacings and dot diameters evoked nearly identical roughness judgments. It was reasoned that these textural properties would challenge any candidate neural coding hypothesis severely by requiring it to account simultaneously for the wide range of roughness magnitudes and the wide differences in surface patterns that evoke the same roughness judgments. The Pacinian responses failed the first test, responding so vigorously and homogeneously to all the surfaces that no conceivable basis for the wide range of subjective magnitudes could be found. Codes based on mean SAI and RA impulse rate failed the second test. Surfaces judged to have the same roughness magnitude evoked widely different discharge rates, and conversely, surfaces that evoked identical mean impulse rates evoked widely different magnitude judgments. In contrast, neural coding measures based on both temporal and spatial variation in SAI and RA firing rates were highly covariant with the magnitude judgments. The results of this study are corroborated by Phillips and Matthews (1993) using a technique based on nerve cooling in humans.

The second study (Connor and Johnson, 1992) used surfaces designed to produce psychophysical results that could only be consistent with the mean impulse rate or a measure of its temporal variation (within-fiber neural codes) or with spatial variation in the impulse rates evoked by the surfaces (a between-fiber code) but not with both. Two series of surfaces were used. In the first series, dot spacing varied from 1.5 to $4 \mathrm{~mm}$ in the direction orthogonal to the scanning direction and remained constant at 4 $\mathrm{mm}$ in the scanning direction. In the second series, the same patterns were rotated by $90^{\circ}$ so that dot spacing varied only in the scanning direction. Because the relationships between a stimulus surface and its SAI and RA neural images are isomorphic in both humans (Phillips et al., 1992) and monkeys (Johnson and Lamb, 1981), the effects of increasing dot spacing on all the relevant response measures were readily predictable. The predictions, which were confirmed by neurophysiological experiments, were as follows: spatial variation in the afferent responses was known to be maximal at dot spacings close to $3.5 \mathrm{~mm}$ from the previous study (Connor et al., 1990); thus, increasing dot spacing in either 
direction from $1.5 \mathrm{~mm}$ toward $4.0 \mathrm{~mm}$ would increase spatial variation.

The effect of increasing dot spacing on mean impulse rate and its temporal variation depends on the pattern orientation. When the dots are closely spaced in one direction and the rows are aligned orthogonal to the scanning direction, each row will evoke a burst of impulses in every afferent fiber, which will contribute strongly to the overall temporal variation in firing rate. When the dot spacing increases, some receptive fields will pass between the dots, fewer impulses will be evoked, and the overall mean rate and its temporal variation will decline. However, when the dots are closely spaced and aligned with the scanning direction, they almost form a continuous line and will evoke a relatively weak and continuous response (i.e., one with little fluctuation in rate). As they move apart, individual dots become more effective stimuli, and the overall mean rate and its fluctuation will increase. Experiments showed that beyond 3.0-3.5 mm the declining number of dots passing each receptive field per unit time becomes the dominant factor, and the mean rate and its fluctuation begin to decline. Thus, increasing dot spacing has opposite effects depending on the orientation of the stimulus patterns.

The result of the psychophysical experiment using these stimuli was that increasing dot spacing led to significant increases in perceived roughness regardless of the pattern orientation, which demonstrates that roughness magnitude could not have been based on temporal or intensive coding measures. Although the neurophysiological experiments confirmed this reasoning, these experiments were not undertaken for that purpose. The lack of any consistent relationship between the roughness judgments and temporal and intensive aspects of the SAI and RA responses was readily predicted from known SAI and RA response properties. Rather, the neurophysiological experiments were undertaken to explore the relationship between roughness perception and spatial variation in the neural image more closely. The neural data showed that mechanisms based on local, directionally specific differences in firing rates produce measures of spatial variation that are closely related to human psychophysical responses (correlation coefficient, 0.984). Center-surround mechanisms yielded significantly lower correlations. So, the second study narrowed the focus to spatial variation in the neural image of the stimulus but provided no significant basis for distinguishing between SAI and RA contributions.

In this, the third study, we have shown that surfaces can be constructed that produce a wide range of roughness judgments and covariant SAI responses but only a narrow range of RA responses that failed the test of consistency. Step-wise regression analyses on the data from this and previous studies suggest that if RA afferent discharge plays any role, it is an inhibitory one. We also showed that the model proposed by Connor and Johnson (1992) accounts for the psychophysical magnitude judgments in all three studies. Thus, our favored hypothesis is that roughness perception is based primarily on spatial variation in the SAI neural image evoked by a textured stimulus.

An issue that requires some discussion is the cross-species assumption inherent in our studies, that neural data derived from the rhesus monkey provide an effective basis for testing hypotheses about the neural mechanisms underlying roughness perception in the human. One approach is to invoke the extensive literature demonstrating the similarity of human (Vallbo, 1995) and monkey (Johnson and Hsiao, 1992) primary afferent responses to a wide variety of mechanical stimuli. However, none of our major conclusions depends on fine quantitative distinctions that might be true in one species but not in the other. Potential neural codes were eliminated only when they were completely inconsistent with the psychophysical data.

Two human psychophysical studies by Lederman and colleagues (Lederman, 1974; Lederman et al., 1982) with no strong assumptions about the underlying neural mechanisms further support the notion that roughness is based on spatial variation in SAI firing rates. One of those studies (Lederman et al., 1982) complements the present study by using stimulus methods that would have affected the RA responses much more strongly than the SAI responses. In that study, intense vibratory adaptation at $20 \mathrm{~Hz}$ had no effect on roughness magnitude judgments although it had a strong effect on vibratory magnitude estimates at $20 \mathrm{~Hz}$ (but not at $250 \mathrm{~Hz}$ ), demonstrating that the RA afferent response was strongly suppressed (Mountcastle et al., 1972; Johnson, 1974). Neurophysiological studies in our own laboratory (Leung et al., 1994) with intense-adapting vibratory stimuli like those used in the experiment of Lederman (1974) confirm this inference and show that the SAI responses would have been relatively unaffected (because they are so much less sensitive to vibratory stimuli than are the RA afferents). The second study by Lederman argues against neural codes based on mean impulse rate or the temporal properties of the response by showing that roughness judgments are affected little by scanning velocity over the range from 10-50 mm/sec (Lederman, 1974; Katz, 1989). In contrast, both temporal and intensive aspects of the response to a textured surface are strongly affected by scanning velocity (Johnson and Lamb, 1981; Phillips et al., 1992). The temporal properties of the response to a scanned stimulus are linked directly to scanning velocity and, thus, are strongly affected. Impulse rates are also affected, increasing significantly with scanning velocity (Johnson and Lamb, 1981; Phillips et al., 1992), whereas roughness judgments are, if anything, diminished by increased scanning velocity (Lederman, 1974). However, spatial variation in the neural image is unaffected as long as the image is isomorphic, which it is over this velocity range (Johnson and Lamb, 1981; Connor et al., 1990; Phillips et al., 1992).

\section{Conclusion}

Analyses presented in this paper show that a single, simple hypothesis based on spatial variation in SAI firing rates accounts for the psychophysical responses to all 47 surfaces used in our three studies; roughness perception can be accounted for by central mechanisms tuned to spatial variation on a scale of 1-3 $\mathrm{mm}$ in the SAI neural image of a textured surface (Figs. 6, 8; Connor and Johnson, 1992). In fact, neurons in area 3b with excitation and inhibition separated by $1-3 \mathrm{~mm}$ have been identified in our laboratory (J. DiCarlo, unpublished observations) using surfaces with randomly arrayed, raised dots. We believe roughness is an example of a percept that varies along an intensive continuum but is coded initially by a spatial mechanism and then is transformed into an intensive neural code, most likely the mean impulse rate of a subpopulation of central neurons with tuned, spatial receptive fields.

The conclusion that SAI afferents are responsible for the perception of tactile roughness adds to the evidence that SAI and RA afferents serve different roles in perception. Roles that have been attributed to SAI afferents include the perception of pressure (Ochoa and Torebjörk, 1983; Mountcastle et al., 1966), spatial form (Johnson and Hsiao, 1992), and tactile roughness. RA afferents have been shown to be responsible for the detection of flutter (Mountcastle et al., 1972; Ochoa and Torebjörk, 1983), 
slip (Johansson and Westling, 1987; Srinivasan et al., 1987), and minute surface elements (LaMotte and Whitehouse, 1986). The hypothesis we favor (Johnson and Hsiao, 1992) is that the two systems are responsible for extracting and processing information about separate aspects of the external world. The existing evidence supports the idea that SAI afferents are responsible for the acquisition of information about form and texture, whereas the RA system is responsible for the detection of flutter, slip, and motion across the skin surface.

\section{REFERENCES}

Blake DT, Hsiao SS, Johnson KO (1997) Slowly and rapidly adapting mechanoreceptive responses to raised and depressed scanned patterns: effects of width, height, orientation, and a raised surround. J Neurophysiol, in press.

Burton H, Sinclair RJ (1994) Representation of tactile roughness in thalamus and somatosensory cortex. Can J Physiol Pharmacol 72:546-557.

Connor CE, Johnson KO (1992) Neural coding of tactile texture: comparisons of spatial and temporal mechanisms for roughness perception. J Neurosci 12:3414-3426.

Connor CE, Hsiao SS, Phillips JR, Johnson KO (1990) Tactile roughness: neural codes that account for psychophysical magnitude estimates. J Neurosci 10:3823-3836.

Johansson RS, Westling G (1987) Signals in tactile afferents from the fingers eliciting adaptive motor responses during precision grip. Exp Brain Res 66:141-154.

Johnson KO (1974) Reconstruction of population response to a vibratory stimulus in quickly adapting mechanoreceptive afferent fiber population innervating glabrous skin of the monkey. J Neurophysiol $37: 48-72$.

Johnson KO (1983) Neural mechanisms of tactual form and texture discrimination. Fed Proc 42:2542-2547.

Johnson KO, Hsiao SS (1992) Neural mechanisms of tactual form and texture perception. Annu Rev Neurosci 15:227-250.

Johnson KO, Hsiao SS (1994) Evaluation of the relative roles of slowly and rapidly adapting afferent fibers in roughness perception. Can J Physiol Pharmacol 72:488-497.

Johnson KO, Lamb GD (1981) Neural mechanisms of spatial tactile discrimination: neural patterns evoked by Braille-like dot patterns in the monkey. J Physiol (Lond) 310:117-144.

Johnson KO, Phillips JR (1988) A rotating drum stimulator for scanning embossed patterns and textures across the skin. J Neurosci Methods 22:221-231.

Johnson KO, Hsiao SS, Blake DT (1996) Linearity as the basic law of psychophysics: evidence from studies of the neural mechanisms of roughness magnitude estimation. In: Somesthesis and the neurobiology of the somatosensory cortex (Franzen O, Johansson RS, Terenius L, eds), pp 213-228. Basel: Birkhäuser.

Katz D (1989) The world of touch (Krueger LE, translator). Hillsdale, NJ: Erlbaum.

LaMotte RH (1977) Psychophysical and neurophysiological studies of tactile sensibility. In: Clothing comfort: interaction of thermal, ventilation construction and assessment factors (Hollies N, Goldman R, eds), pp 83-105. Ann Arbor, MI: Ann Arbor Science.

LaMotte RH, Whitehouse JM (1986) Tactile detection of a dot on a smooth surface: peripheral neural events. J Neurophysiol 56:1109-1128.

Lederman SJ (1974) Tactile roughness of grooved surfaces: the touching process and the effects of macro- and microsurface structure. Percept Psychophys 16:385-395.

Lederman SJ, Loomis JM, Williams DA (1982) The role of vibration in the tactual perception of roughness. Percept Psychophys 32:109-116.

Leung YY, Hsiao SS, Johnson KO (1994) Adaptation of mechanoreceptive afferents to continuous sinusoidal vibration. Soc Neurosci Abstr 20:1387.

Mountcastle VB, Talbot WH, Kornhuber HH (1966) The neural transformation of mechanical stimuli delivered to the monkey's hand. In: Touch, heat, pain and itch (de Reuck AVS, Knight J, eds), pp 325-351. London: Churchill.

Mountcastle VB, LaMotte RH, Carli G (1972) Detection thresholds for stimuli in humans and monkeys: comparison with threshold events in mechanoreceptive afferent nerve fibers innervating the monkey hand. J Neurophysiol 35:122-136.

Ochoa JL, Torebjörk HE (1983) Sensations evoked by intraneural microstimulation of single mechanoreceptor units innervating the human hand. J Physiol (Lond) 342:633-654.

Phillips JR, Matthews PBC (1993) Texture perception and afferent coding distorted by cooling the human ulnar nerve. J Neurosci 13:2332-2341.

Phillips JR, Johansson RS, Johnson KO (1992) Responses of human mechanoreceptive afferents to embossed dot arrays scanned across fingerpad skin. J Neurosci 12:827-839.

Sathian K, Goodwin AW, John KT, Darian-Smith I (1989) Perceived roughness of a grating: correlation with responses of mechanoreceptive afferents innervating the monkey's fingerpad. J Neurosci 9:1273-1279.

Srinivasan MA, Whitehouse JM, LaMotte RH (1987) Detection of slip: peripheral neural coding. J Neurosci 7:1682-1697.

Talbot WH, Darian-Smith I, Kornhuber HH, Mountcastle VB (1968) The sense of flutter-vibration: comparison of the human capacity with response patterns of mechanoreceptive afferents from the monkey hand. J Neurophysiol 31:301-355.

Twombly IA, DiCarlo JJ, Johnson KO, Hsiao SS (1996) Linear and non-linear processing of spatial form in area $3 \mathrm{~b}$ of the awake monkey. Soc Neurosci Abstr 22:18.

Vallbo ÅB (1995) Single-afferent neurons and somatic sensation in humans. In: The cognitive neurosciences (Gazzaniga MS, ed), pp $237-$ 252. Cambridge, MA: MIT. 